\title{
Histone deacetylase inhibitors protect against cisplatin-induced acute kidney injury by activating autophagy in proximal tubular cells
}

Jing Liư ${ }^{1,2}$, Man J. Livingston², Guie Dong ${ }^{2}$, Chengyuan Tang ${ }^{1}$, Yunchao $\mathrm{Su}^{3}$, Guangyu $\mathrm{Wu}^{3}$, Xiao-Ming Yin ${ }^{4}$ and Zheng Dong ${ }^{1,2}$

\begin{abstract}
Histone deacetylase inhibitors (HDACi) have therapeutic effects in models of various renal diseases including acute kidney injury (AKI); however, the underlying mechanism remains unclear. Here we demonstrate that two widely tested HDACi (suberoylanilide hydroxamic acid (SAHA) and trichostatin A (TSA)) protect the kidneys in cisplatin-induced AKI by enhancing autophagy. In cultured renal proximal tubular cells, SAHA and TSA enhanced autophagy during cisplatin treatment. We further verified the protective effect of TSA against cisplatin-induced apoptosis in these cells. Notably, inhibition of autophagy by chloroquine or by autophagy gene 7 (Atg7) ablation diminished the protective effect of TSA. In mice, TSA increased autophagy in renal proximal tubules and protected against cisplatin-induced AKI. The in vivo effect of TSA was also abolished by chloroquine and by Atg7 knockout specifically from renal proximal tubules. Mechanistically, TSA stimulated AMPK and inactivated mTOR during cisplatin treatment of proximal tubule cells and kidneys in mice. Together, these results suggest that HDACi may protect kidneys by activating autophagy in proximal tubular cells.
\end{abstract}

\section{Introduction}

Histone deacetylases (HDACs) are important players in epigenetic regulation that catalyze the removal of acetyl groups from $\varepsilon$-N-acetyl lysine residues on histones, resulting in an increase of positive charges in histones, tight DNA binding, and compact chromatin to repress gene transcription ${ }^{1}$. HDACs may also deacetylate nonhistone proteins. As such, HDACs play crucial roles in diverse biological processes and deregulation of HDACs contributes to the pathogenesis of major diseases ${ }^{2}$. The

\footnotetext{
Correspondence: Zheng Dong (zdong@csu.edu.cn) (zdong@augusta.edu) 'Department of Nephrology, The Second Xiangya Hospital, Central South University, Changsha, Hunan 410011, China

${ }^{2}$ Department of Cellular Biology \& Anatomy, Medical College of Georgia at Augusta University and Charlie Norwood VA Medical Center, Augusta, GA 30912, USA

Full list of author information is available at the end of the article Edited by G.M. Fimia
}

research of HDACs has also led to the development of HDAC inhibitors (HDACis) therapeutic effects ${ }^{3}$. In kidneys, HDACis have therapeutic potentials in experimental models of renal fibrosis ${ }^{4-6}$, acute kidney injury $(\mathrm{AKI})^{7-9}$, polycystic kidney disease ${ }^{10,11}$, diabetic nephropathy ${ }^{12,13}$, and HIV-associated nephropathy ${ }^{14}$. The mechanisms underlying the beneficial effect of HDACis in kidneys remain poorly understood ${ }^{15,16}$.

We demonstrated dose-dependent effects of HDACi in renal tubular cells ${ }^{8,17}$. At micromolar concentrations, suberoylanilide hydroxamic acid (SAHA) induced cell death in these cells, whereas at lower doses SAHA protected the cells from cisplatin-induced apoptosis. Cisplatin, a potent cancer therapy drug, has notorious side effects in normal tissues, especially in kidneys ${ }^{18-22}$. A pathological hallmark of cisplatin nephrotoxicity is cell injury and death in renal tubules $^{23}$. Interestingly, HDACis, including both SAHA and 
trichostatin A (TSA), may suppress cisplatin-induced tubular cell apoptosis and AKI in mice ${ }^{7-9}$, but the underlying mechanism is largely unclear.

Autophagy is a highly conserved lysosomal degradation pathway that eliminates protein aggregates and dysfunctional organelles ${ }^{24,25}$. In cellular stress, autophagy is activated and serves primarily as an adaptive mechanism for cell survival, whereas deregulated autophagy plays important roles in the pathogenesis of various diseases ${ }^{26,27}$. In ischemic and nephrotoxic AKI, autophagy is induced to protect against tubular cell injury and death ${ }^{28-31}$. The current study tested the hypothesis that HDACis may protect kidney cells and tissues by upregulating autophagy. We demonstrated that both SAHA and TSA enhanced autophagy in renal tubular cells. TSA further protects against cisplatin-induced injury in renal tubular cells and AKI in mice, and notably, the protective effects of TSA were diminished by pharmacological and genetic inhibition of autophagy.

\section{Results}

Autophagy is induced by TSA and SAHA in renal proximal tubular cells (RPTCs)

Treatment of RPTCs with TSA led to LC3B-II accumulation, a biochemical hallmark of autophagy ${ }^{32}$ (Fig. 1a: lanes 3, 5, 7 vs lane 1; lanes 4, 6, 8 vs lane 2). LC3B-II accumulation was markedly increased by chloroquine, an auto-lysosomal inhibitor ${ }^{32}$ (Fig. 1a: lanes 2, 4, 6, 8 vs lanes $1,3,5,7)$. The effect of chloroquine appeared more in TSA-treated cells (lane 2 vs lanes 4, 6, 8), indicating an increased LC3B-II turnover and induction of autophagic flux by TSA. We then examined the expression of LC3BII at two different time points of 8 and $20 \mathrm{~h}$. There was an early LC3B-II accumulation following $8 \mathrm{~h}$ of TSA treatment (Fig. 1d, lane 3 vs lane 1; lane 4 vs lane 2), accompanied with an increased LC3B-II turnover (lanes 2, 4 vs lanes 1,3$)$. Consistently, these changes were maintained at $20 \mathrm{~h}$ of TSA treatment (lanes 5,6 vs lanes 1,2 ). The immunoblotting results were confirmed by densitometry of LC3B-II signals (Fig. 1b, c, e, f). Similarly, SAHA, another pan-HDACi, also induced autophagy in RPTC cells as indicated by LC3B-II (Supplementary Figure 1). We further monitored autophagic dynamics by transfecting monomeric red fluorescent protein (mRFP)-green fluorescent protein (GFP)-LC3 plasmids. In control cells, both green GFP-LC3 and red RFP-LC3 signals were mostly diffuse in cells (Fig. 1g, CON). Following TSA treatment, numerous GFP-LC3 and RFP-LC3 puncta appeared (Fig. 1g, TSA). Almost all GFP signals colocalized with RFP, exhibiting a yellow color indicative of autophagosomes. Notably, the acid-sensitive GFP fluorescence faded or disappeared upon fusion with lysosomes, whereas the acid-insensitive RFP signals were maintained. Therefore, a portion of RFP-LC3 puncta with no or very weak GFP signals showed orange-red staining in overlapping images, which represented autolysosomes (Fig. 1g, TSA). Quantitatively, the number of autophagosomes per cell was increased from $\sim 9$ in control cells to $\sim 55$ in TSAtreated cells. The number of autolysosomes per cell was also remarkably elevated from $\sim 2$ in control to $\sim 61$ in TSA-treated cells (Fig. 1h). We further calculated the proportion of autolysosomes in the total number of RFPLC3 puncta to indicate autophagic flux ${ }^{32}$. Control cells had a basal rate of $\sim 17 \%$ autophagic flux, which was increased to $\sim 54 \%$ by TSA (Fig. 1i).

\section{TSA enhances autophagy during cisplatin treatment of proximal tubular cells}

We further examined the effects of TSA on cisplatininduced autophagy in RPTC cells. Cisplatin treatment for $20 \mathrm{~h}$ led to a moderate yet significant accumulation of LC3B-II (Fig. 2a, b: lanes 3, 4 vs lanes 1, 2), which was further enhanced by TSA (lanes 7,8 vs lanes 3,4 ). LC3BII turnover assay in Fig. 2c also showed that control cells had a 1.68-fold basal level of turnover, which was increased in cisplatin-treated cells (2.08-fold) but significantly promoted by cisplatin+TSA (2.6-fold). In the cells expressing mRFP-GFP-LC3 (Fig. 2d-f), cisplatin treatment increased the numbers of both GFP-LC3 and RFP-LC3 puncta. Notably, the average numbers of autophagosomes and autolysosomes per cell in cisplatinonly group were $\sim 25$ and $\sim 16$, which were increased to $\sim 58$ and $\sim 65$, respectively, in the cisplatin+TSA group (Fig. 2e). Consistently, cisplatin-induced autophagic flux was also elevated from $\sim 39 \%$ to $\sim 53 \%$ by TSA (Fig. 2 f), indicating that TSA further enhances cisplatin-induced autophagy in RPTC cells.

\section{Inhibition of autophagy by chloroquine eliminates the protective effects of TSA in cisplatin-treated RPTC cells}

To determine the involvement of autophagy in the protective effect of HDACi, we first compared the effects of TSA on cisplatin-induced tubular cell apoptosis in the absence vs presence of chloroquine. In the absence of chloroquine, cisplatin treatment for $20 \mathrm{~h}$ led to $\sim 50 \%$ apoptosis in RPTC cells, which was partially but significantly reduced to $\sim 30 \%$ by TSA (Fig. 3a: without CQ, Fig. 3b). Consistently, cisplatin-induced caspase activation was also attenuated by TSA under these conditions (Fig. 3c). In sharp contrast, the beneficial role of TSA disappeared when autophagy was inhibited by chloroquine. Consistent with our previous studies ${ }^{33,34}$, cisplatin-induced apoptosis in RPTC cells was worsened by chloroquine. Notably, in the presence of chloroquine, TSA was unable to suppress cisplatin-induced apoptosis and caspase activation (Fig. 3a: with CQ, Fig. 3d, e), suggesting a role of autophagy in the cytoprotective effects of TSA. 


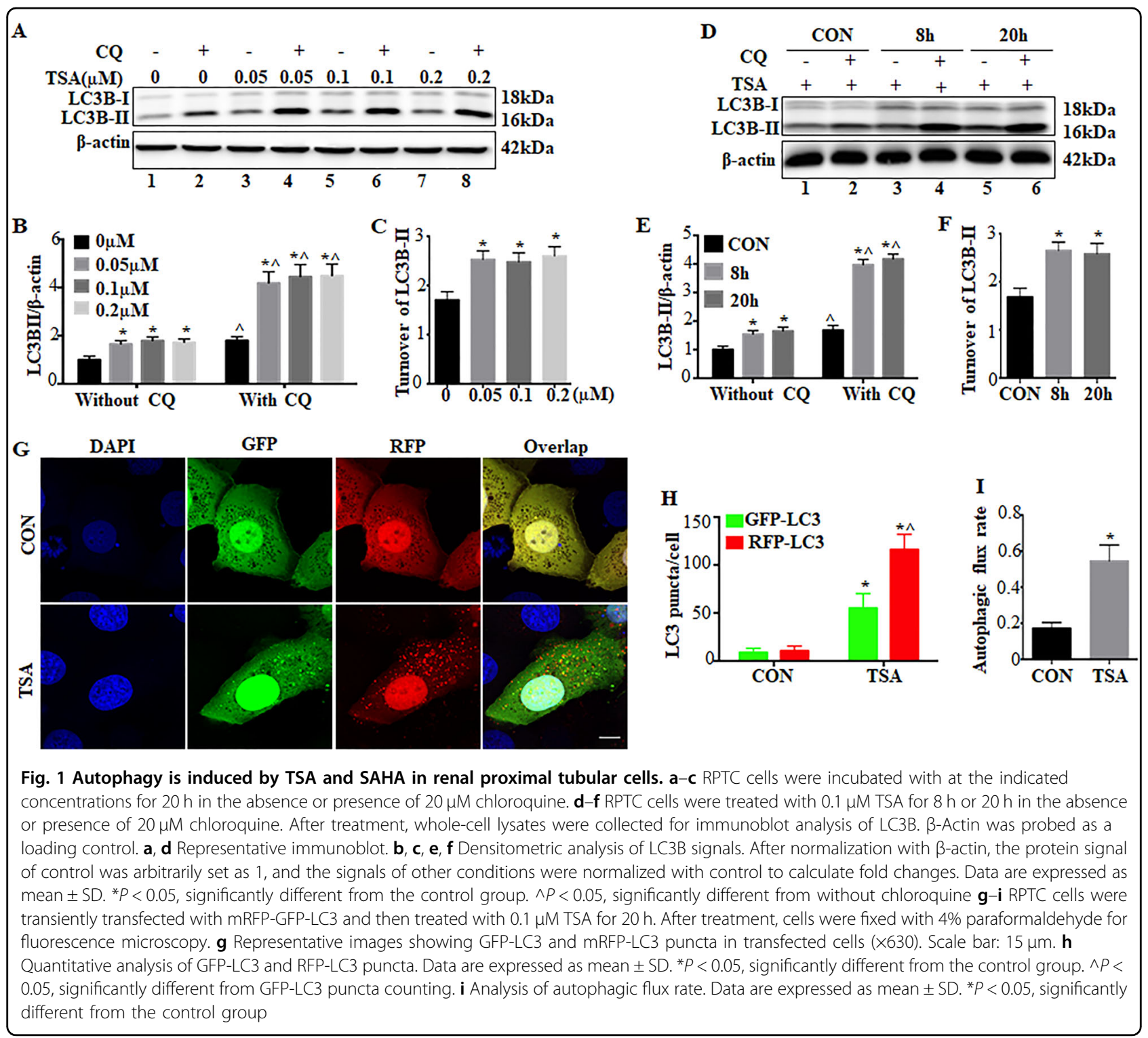

The cytoprotective effect of TSA is abrogated in Atg7-KO mouse kidney proximal tubular cells

We further compared the effects of TSA in Atg7-KO and wild-type (WT) mouse proximal tubular cells (MPTCs) generated in our recent work ${ }^{35}$. We verified autophagy deficiency in Atg7-KO cells by transfecting mRFP-GFP-LC3 (Fig. 4a-d). In control WT cells. cisplatin induced the formation of autophagosomes and autolysosomes and an increase of autophagic flux. TSA activated autophagy on its own, and importantly, it further promoted cisplatin-induced autophagy in WT MPTC cells (Atg7 WT, TSA, CIS+TSA). However, these autophagic responses were remarkably impaired in Atg7-KO MPTC cells (Fig. 4a-d, Atg7 KO). GFP-LC3 puncta were rarely seen in these knockout (KO) cells. Under both control and cisplatin-treated conditions, the numbers of RFP-LC3 puncta and autophagic flux rate were largely reduced in Atg7 KO cells as compared with WT cells. Furthermore, the autophagy-stimulating effects of TSA (either used alone or with cisplatin) were also abrogated in Atg7 KO cells. These morphological observations were further confirmed by immunoblot analysis of ATG7 and LC3B (Fig. 4e, f). In WT cells, cisplatin induced a $\sim 1.7$-fold LC3B-II accumulation, which was further increased to 2.7-fold by TSA. By contrast, there was no detectable ATG7 expression in Atg7 $\mathrm{KO}$ cells, and as a result, the conversion of LC3B-I to LC3B-II was almost completely attenuated.

We next examined the effects of autophagy deficiency in Atg7 KO MPTC cells on cisplatin-induced apoptosis. Both 


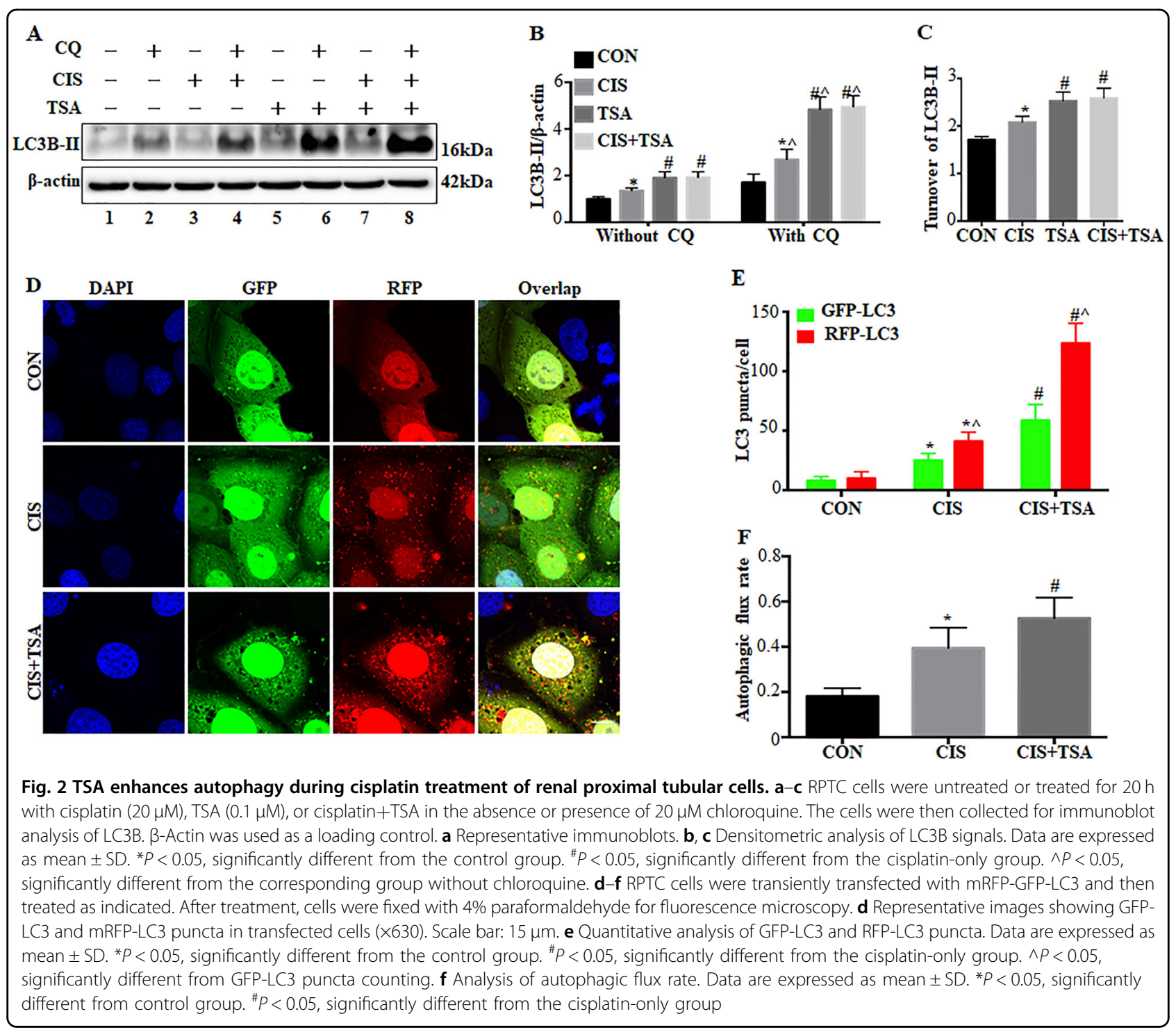

WT and Atg7-KO cells had minimal apoptosis under control conditions or TSA-only treatment (Fig. 5a, b). Cisplatin induced $\sim 30 \%$ apoptosis in WT cells, which was reduced to $\sim 16 \%$ by TSA. Notably, cisplatin induced $\sim 46 \%$ apoptosis in Atg7 KO cells, which was only slightly suppressed by TSA to $41 \%$ (Fig. 5a, b), indicating the protective effects of TSA were abolished in Atg7 KO cells. By calculation, the inhibitory efficiency of TSA was $46 \%$ in WT cells and $11 \%$ in ATG7 KO cells (Fig. 5c). Consistently, TSA significantly suppressed caspase activation during cisplatin treatment in WT cell, but it was much less effective in Atg7-KO cells (Fig. 5d, e). Together with the choloquine results, these data provide convincing evidence that autophagy plays a critical role in the cytoprotective role of TSA.
TSA enhances renal tubular autophagy during cisplatin treatment in mice

We then examined the effects of TSA on tubular autophagy in a mouse model of cisplatin-induced AKI. As previously $^{33,34}$, a single dose of cisplatin at $30 \mathrm{mg} / \mathrm{kg}$ induced a persistent upregulation of LC3B-II in kidneys for up to 4 days (Fig. 6a: lanes 2, 4, 6 vs lane 1). TSA daily injection at $1 \mathrm{mg} / \mathrm{kg}$ further enhanced LC3B-II accumulation in cisplatin-treated $\mathrm{C} 57 \mathrm{Bl} / 6$ mice (Fig. 6a: lanes 3, 5, 7 vs lanes $2,4,6)$. These results were substantiated by densitometric analysis of LC3B-II signals (Fig. 6b). Autophagy induction by cisplatin was further shown by immunohistochemical staining of LC3B (Fig. 6c), which showed a granular, punctate staining in proximal tubule cells during cisplatin treatment. Of note, TSA further 


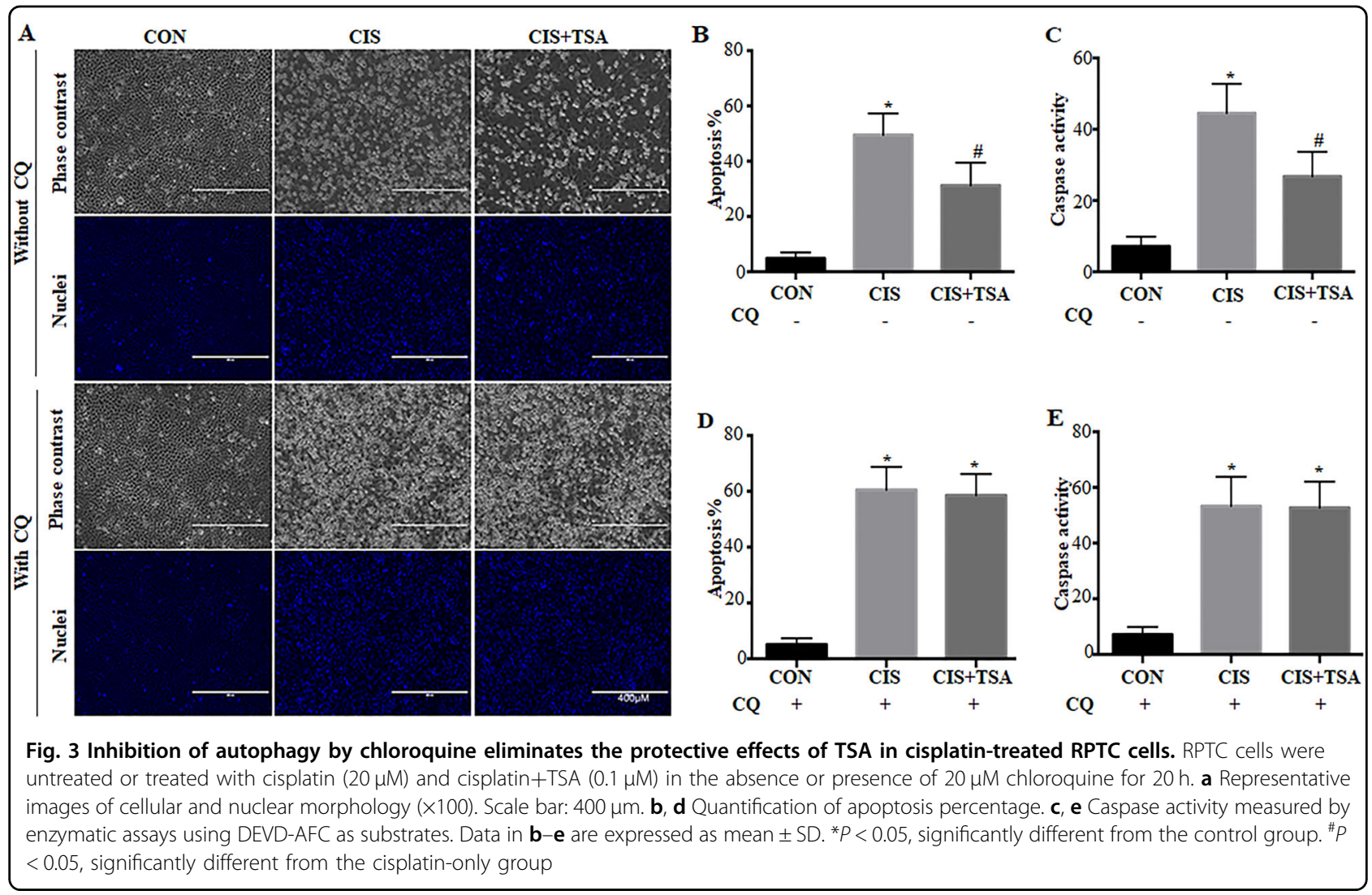

enhanced LC3B punctate staining in both numbers and intensity. Quantitatively, the number of LC3B-positive puncta per proximal tubule was increased from $\sim 4$ in saline control kidneys to $\sim 10$ in cisplatin-treated mice and further to $\sim 23$ in the cisplatin+TSA group (Fig. 6d). To determine the dynamic changes of autophagy in kidneys, we tested the autophagy reporter mouse model that expresses the CAG-RFP-GFP-LC3 transgene ${ }^{36}$. As shown in Fig. 6e, the majority of renal tubules in control kidneys had few GFP-LC3 puncta, while a small percentage of tubules had RFP-LC3 puncta at relatively low intensity at the apical side toward the lumen. Cisplatin treatment led to increases in the numbers of both GFP-LC3 and RFPLC3 puncta in proximal tubules, which were further increased by TSA. Under these conditions, the punctate LC3 staining, particularly RFP-LC3 puncta, accumulated intensively around the nuclei in proximal tubular cells (Fig. 6e). We further calculated the numbers of autophagosomes and autolysosomes as well as autophagic flux rate in vivo (Fig. 6f, g). Saline control mice had $\sim 11$ autophagosomes (GFP-LC3 and RFP-LC3 co-staining puncta) per proximal tubule, which was increased to $\sim 26$ by cisplatin and further to $\sim 48$ by cisplatin + TSA. The number of autolysosomes (RFP-LC3 puncta without GFPLC3 signal) per proximal tubule was also increased from $\sim 6$ in saline control to $\sim 21$ in cisplatin-treated kidneys and further to $\sim 70$ in the cisplatin+TSA group (Fig. $6 \mathrm{f}$ ).
Accordingly, autophagic flux rate was increased from $\sim 31 \%$ in saline control to $\sim 44 \%$ by cisplatin and further to $\sim 59 \%$ by cisplatin+TSA (Fig. $6 \mathrm{~g}$ ). Together, these results suggest that TSA promotes cisplatin-induced autophagy in proximal tubules in mice.

\section{Inhibition of autophagy by chloroquine abolishes the} protective effects of TSA in cisplatin-induced AKI in mice

To delineate the role of autophagy in vivo, we examined chloroquine on the renoprotective effects of TSA in cisplatin-treated $\mathrm{C} 57 \mathrm{Bl} / 6$ mice. Cisplatin treatment led to AKI as indicated by increased serum creatinine (Fig. 7a). TSA suppressed cisplatin-induced serum creatinine from $\sim 1.1$ to $\sim 0.7 \mathrm{mg} / \mathrm{dl}$ (CIS vs CIS+TSA). Consistenly, cisplatin-induced tubular damage in renal cortex and out medulla was also inhibited by TSA. Hematoxylin-eosin $(\mathrm{H}-\mathrm{E})$ staining revealed that cisplatin induced histopathological damages of kidney tissues, which were ameliorated by TSA (Fig. 7b: CIS vs CIS+TSA). In quantification, tubular damage score was reduced from $\sim 3$ in the cisplatin group to $\sim 2$ in the cisplatin+TSA group (Fig. 7c: CIS vs CIS+TSA). Cisplatin also induced caspase activation and tubular apoptosis as indicated by cleaved caspase 3, which was also attenuated by TSA (Fig. 7d: CIS vs CIS+TSA). However, in the presence of chloroquine, the protective effects of TSA were completely compromised (Fig. $7 \mathrm{a}-\mathrm{d}$ : CIS+CQ vs CIS+TSA 


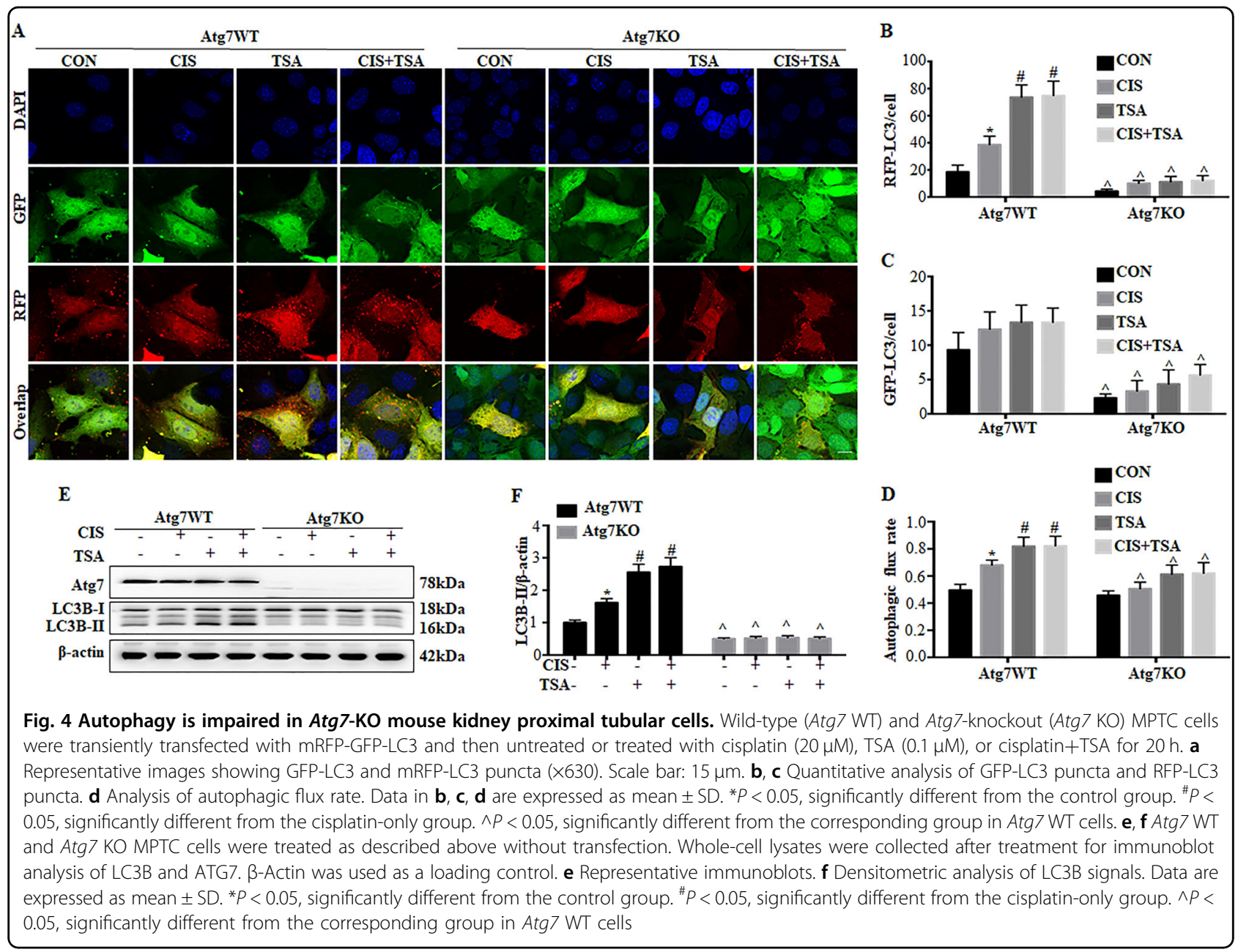

$+\mathrm{CQ}$ ), indicating that autophagy induction is involved in the renoprotective effects of TSA.

\section{The renoprotective effect of TSA in cisplatin-induced AKI is} lost in PT-Atg7 KO mice

We further determined whether the renoprotective effect of TSA depends on autophagy by testing the conditional autophagy-deficient mouse model with Atg7 specifically deleted from kidney proximal tubules (PT$\operatorname{Atg} 7 \mathrm{KO})^{34,35}$. In WT control (PT-Atg7 WT) mice, cisplatin led to LC3B-II accumulation in kidney tissues on day 3. This upregulation of LC3B-II was further enhanced by TSA (Fig. 8a, b: PT-Atg7 WT). In contrast, the accumulation of LC3B-II were largely inhibited in PT-Atg7 KO kidneys, indicating autophagy deficiency in these mice (Fig. 8a, b: PT-Atg7 KO). In immunohistochemical analysis, cisplatin induced a punctate LC3B staining in proximal tubules and the intensity of LC3B-positive puncta was further elevated by TSA in PT-Atg7 WT mice (Fig. 8c: PT-Atg7 WT). Quantitatively, in PT-Atg7 WT mice there were $\sim 6 \mathrm{LC} 3 \mathrm{~B}$-positive puncta per tubule in the saline control group, which was increased to $\sim 13$ by cisplatin and further to $\sim 23$ in the cisplatin+TSA group (Fig. 8d: PT-Atg7 WT). In contrast, PT-Atg7 KO mice mainly showed diffuse LC3B staining in proximal tubules (Fig. 8c, d: PT-Atg7 KO).

We further monitored kidney injury. In WT mice, cisplatin induced increases in both serum creatinine and blood urea nitrogen (BUN) (Fig. 9a, c: PT-Atg7 WT, CIS). TSA partially but significantly suppressed the loss of renal function. Serum creatinine and BUN were reduced from 1.9 and $158 \mathrm{mg} / \mathrm{dl}$ in the cisplatin group to 1.2 and 119 $\mathrm{mg} / \mathrm{dl}$ in the cisplatin+TSA group, respectively (Fig. 9a, c: PT-Atg7 WT, CIS vs CIS+TSA). Consistently, TSA also ameliorated cisplatin-induced tubular damage in renal cortex and out medulla, as indicated by histological examination. Cisplatin-treated mice had a tubular damage score of 3, which was reduced to 1.9 by TSA (Fig. 9e, f: PT-Atg7 WT, CIS vs CIS+TSA). Cisplatin-induced caspase activation was also attenuated by TSA (Fig. 9g, h: PTAtg7 WT, CIS vs CIS+TSA). We then determined the effects of TSA in PT-Atg7 KO mice with impaired 


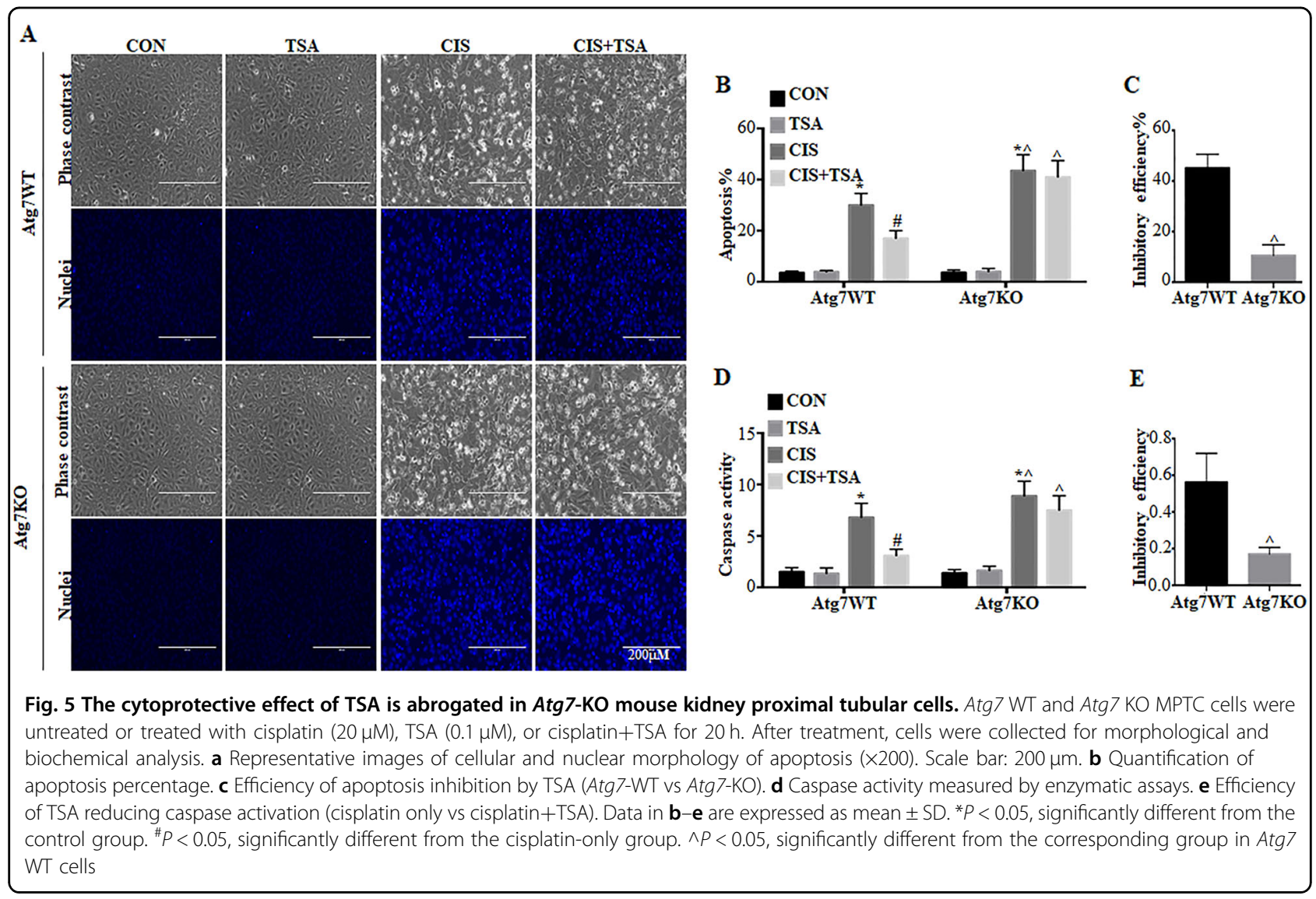

autophagy in proximal tubules. Consistent with previous work $^{34}$, PT-Atg7 KO mice developed more severe kidney injury following cisplatin treatment than their WT littermates (Fig. 9a, c, e-h: WT vs KO, CIS). Notably, TSA only had marginal inhibitory effects on cisplatin-induced renal function loss in PT-Atg7 KO mice (Fig. 9a, c: PTAtg7 KO, CIS vs CIS+TSA). The inhibition efficiency of TSA on serum creatinine and BUN in WT mice was $25-35 \%$, which was decreased to $\sim 10 \%$ in PT-Atg7 KO mice (Fig. 9b, d). Moreover, TSA did not have obvious effects on cisplatin-induced tubular cell injury and death in PT-Atg7 KO mice (Fig. 9e-h: PT-Atg7 KO, CIS vs CIS +TSA). These results, together with the chloroquine test (Fig. 7), provide compelling in vivo evidence that TSA protects against cisplatin-induced AKI by activating autophagy in proximal tubules.

TSA activates AMPK and inactivates mammalian target of rapamycin (mTOR) during cisplatin treatment of RPTC cells and $\mathrm{C} 57 \mathrm{BI} / 6$ mice

Autophagy involves a complex molecular machinery that is regulated at various levels by multiple signaling pathways $^{24,25}$. To gain initial insights into the mechanism by which TSA activates autophagy in proximal tubular cells, we examined AMPK and mTOR, two well- recognized autophagy regulatory pathways ${ }^{24,25,37}$. Cisplatin induced phosphorylation/activation of AMPK in RPTC cells, which was accompanied with marginal inactivation of mTOR as indicated by decrease in phosphorylated P70S6K (p-P70S6K) (Fig. 10a-c). TSA, either used alone or with cisplatin, significantly increased $\mathrm{p}$ AMPK and decreased p-P70S6K, suggesting AMPK activation and mTOR inactivation by TSA (Fig. 10a-c). Similar effects of TSA were observed in mouse kidney tissues (Fig. 10d-e). These results suggest that TSA may enhance autophagy in renal tubular cells to protect kidneys by activating AMPK and suppressing mTOR.

\section{Discussion}

Recent studies have demonstrated the therapeutic effects of HDACis in several experimental models of kidney diseases, but the underlying mechanisms remain largely unclear. The current study has further confirmed the protective effects of HDACi in cisplatin-induced nephrotoxicity. Importantly, we show that TSA and SAHA, two classical HDACis, can stimulate autophagy in RPTCs and kidneys during cisplatin treatment. Inhibition of autophagy either by chloroquine or by $\operatorname{Atg} 7$ knockout in proximal tubular cells led to the attenuation of the renoprotective effects of TSA, supporting a critical role of 


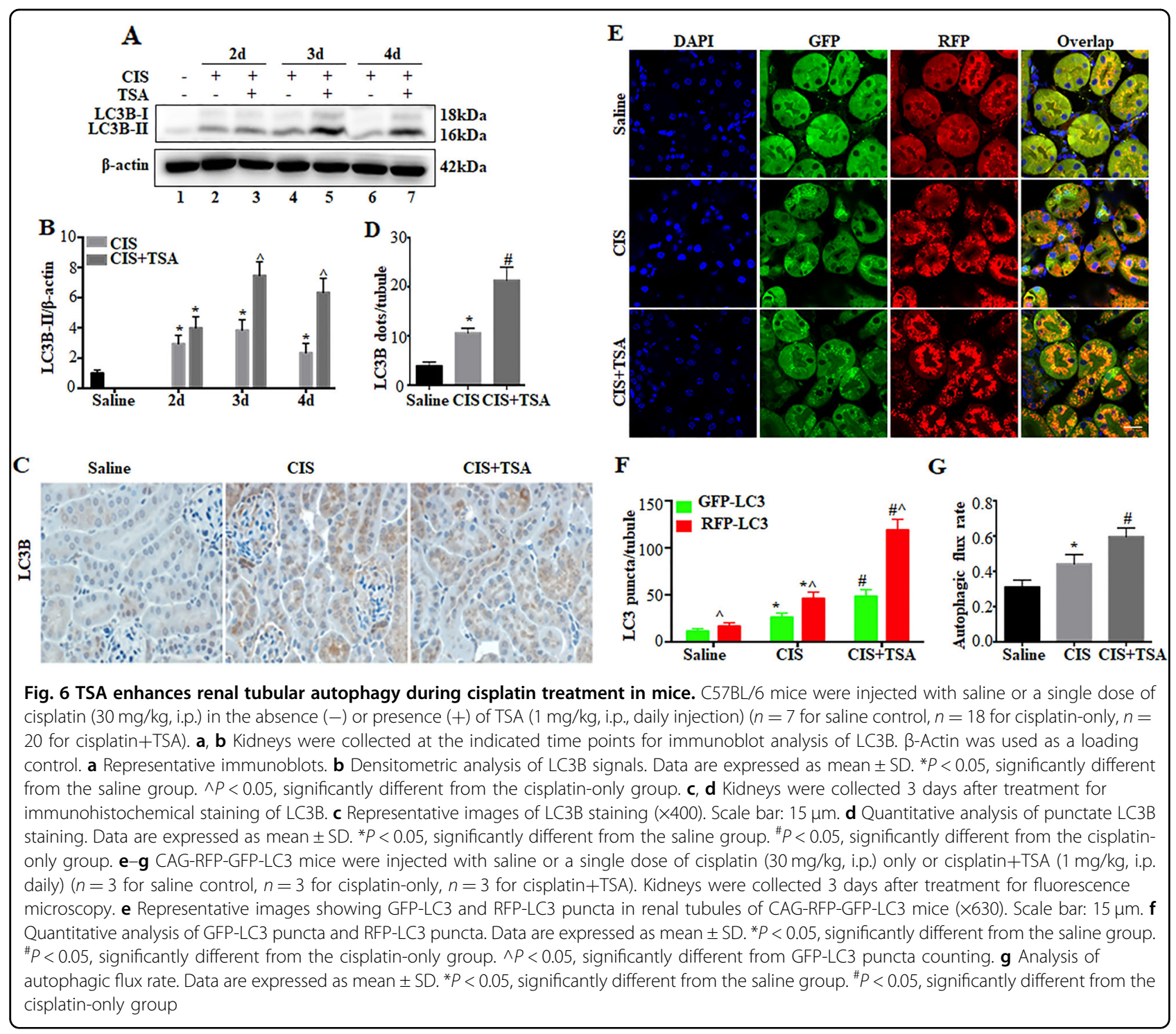

autophagy in the protective action of TSA. Mechanistically, TSA stimulated AMPK and inactivated mTOR during cisplatin treatment, suggesting that HDACis may enhance autophagy by activating AMPK and inhibiting mTOR, two major regulators of autophagy ${ }^{24,25,37}$.

Arany et al. ${ }^{7}$ demonstrated the cytoprotective effects of TSA against cisplatin-induced apoptosis in mouse RPTCs. We further verified protective effects of SAHA and TSA in rat $\mathrm{RPTCs}^{8}$. Notably, these agents also increased cell recovery from cisplatin cytotoxicity, indicating a longterm pro-survival effect ${ }^{8}$. Mechanistically, we showed that SAHA and TSA could block the DNA damage response during cisplatin treatment, resulting in the suppression of p53 activation, a major signaling pathway for cisplatininduced tubular cell apoptosis ${ }^{8}$. Ramesh and colleagues ${ }^{9}$ further revealed the anti-inflammation effects of HDACis in cisplatin nephrotoxicityby transcriptional induction of activated microglia/macrophage WAP domain protein. Our current study has identified autophagy as a new mechanism responsible for HDACi-mediated renoprotection during cisplatin nephrotoxicity. In our experiments, both TSA and SAHA induced autophagy in proximal tubular cells (Fig. 1, Supplementary Figure 1). TSA further enhanced autophagy during cisplatintreatment of proximal tubular cells and kidneys (Fig. 2 and Fig. 6). These morphological and biochemical analyses confirmed autophagy induction and enhancement by HDACis at steady-state levels and, more importantly, revealed the dynamic regulation of autophagy by these agents. To define the role of autophagy, we used both pharmacological and genetic approaches. In cultured proximal tubular cells, impairment of autophagy either by chloroquine or $\operatorname{Atg} 7$ knockout significantly attenuated the inhibitory effects of TSA on cisplatin-induced apoptosis 


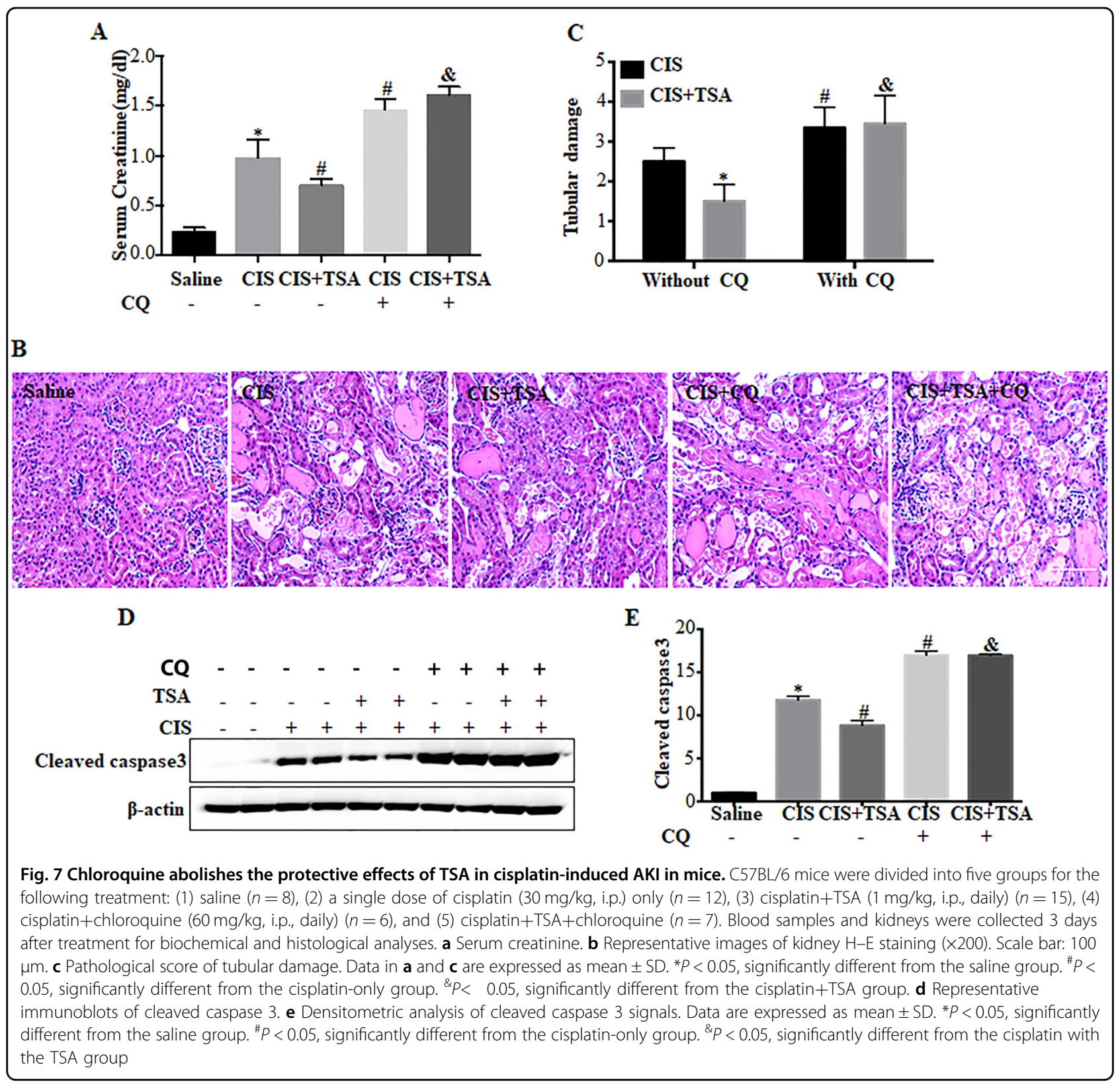

(Figs. 3 and 5). Consistently, TSA lost its renoprotective effects in mice when autophagy was blocked by chloroquine or Atg7 ablation from kidney proximal tubules (Figs. 7 and 9). These results unequivocally demonstrate the importance of autophagy to the protective effects of HDACi in cisplatin nephrotoxicity. Interestingly, impairment of autophagy may also delays kidney recovery from ischemia-reperfusion injury ${ }^{38}$, suggesting a role of autophagy in cell or tissue recovery from injury.

A few recent studies have eluded the possible regulation of autophagy by HDACi. Patschan et al. demonstrated that SAHA could abrogate the autophagy inhibitory effect of transforming growth factor- $\beta$ in endothelial progenitor cells $^{39}$. Khan et al. showed that HDACi may prevent the decline of podocyte autophagy in diabetic models and attenuate podocyte injury as well as proteinuria ${ }^{40}$. SAHA was also shown to increase autophagic flux in rabbit myocardium and protect against ischemia/reperfusion injury ${ }^{41}$, while TSA may promote autophagy in neurons and ameliorate neuronal apoptosis and brain injury induced by subarachnoid hemorrhage in rats ${ }^{42}$. Despite these studies, the role of autophagy in the effects of HDACis remains unclear. Using pharmacologic and genetic inhibitory approaches, our current study has shown convincing evidence that autophagy is critical to the renoprotective effects of HDACi in cisplatin nephrotoxicity. 


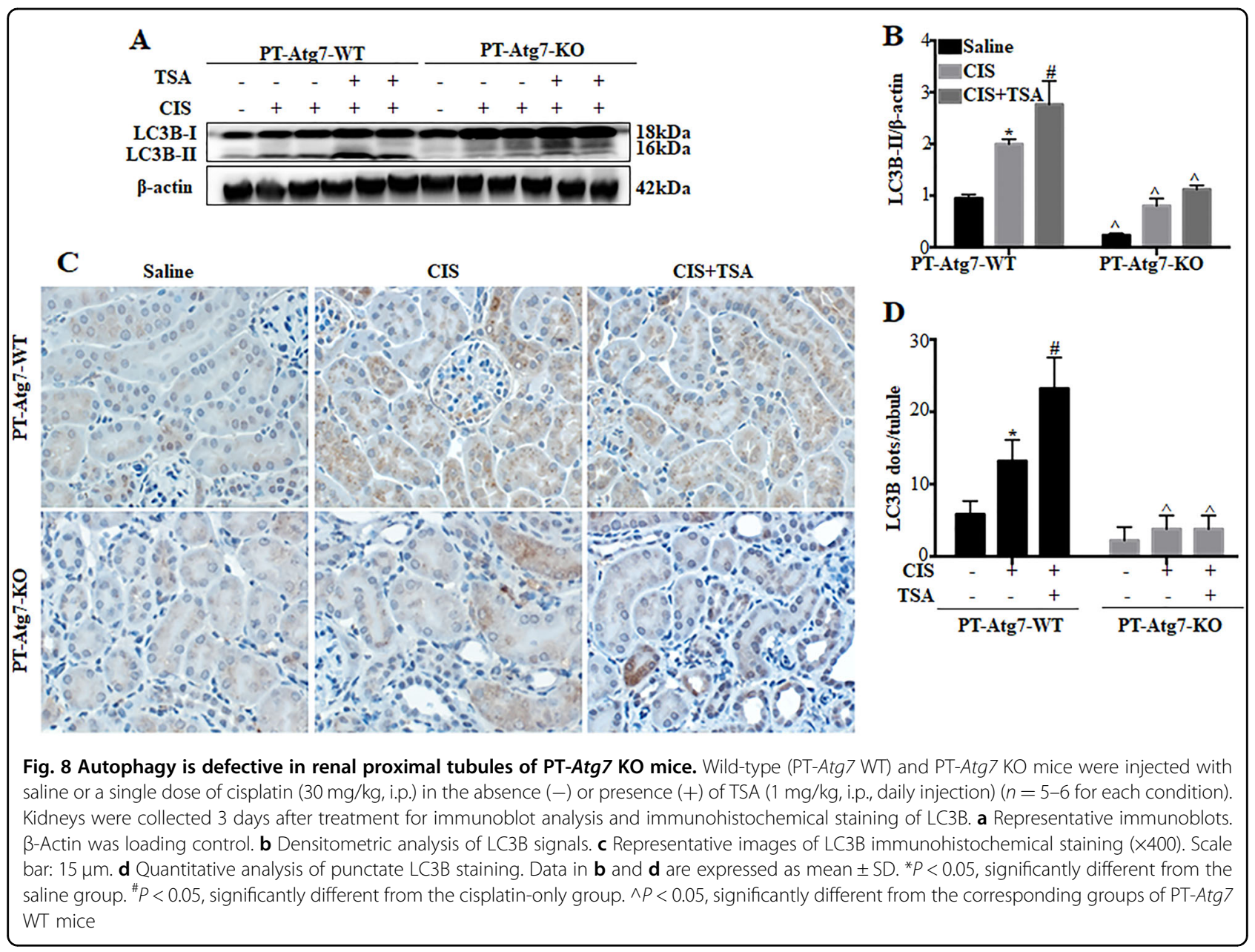

As a promising class of anticancer agents, HDACi have synergistic or additive tumor-killing effects with many other anticancer drugs such as cisplatin ${ }^{43}$. Using HDACi to prevent renal damage without affecting the efficacy of cisplatin in cancer treatment appears to be a very attractive therapeutic strategy. In this regard, our previous study showed that SAHA, while reducing cisplatin-induced apoptosis in kidney tubular cells, could enhance apoptosis during cisplatin treatment of HCT116 colon cancer cells ${ }^{8}$, supporting the possibility of in vivo use of HDACis for renoprotection while enhancing the tumor-killing effects of cisplatin. Although the death-promoting mechanisms of SAHA in HCT116 cancer cells remain unclear, the results indicate that normal cells and cancer cells may respond differently to $\mathrm{HDACis}^{8}$. Indeed, in cancer models of hepatocellular carcinoma, endometrial stromal sarcoma, pancreatic cancer, and ovarian cancer, HDACis can induce autophagy to elicit both apoptotic and nonapoptotic cell death ${ }^{3}$. Given these findings, HDACiinduced autophagy in normal renal tubular cells may represent a new kidney-specific protective strategy during cisplatin-mediated chemotherapy. Kidney tubular cells may have significantly higher endonuclease and caspase activities than cancer cells ${ }^{44,45}$, which may contribute to the sensitivity of kidney tissues to HDACi protection.

The mechanisms whereby HDACis regulate autophagy remain largely unclear. HDAC1, HDAC2, HDAC6, Sirt3, and Sirt6 were recently shown to increase at $24 \mathrm{~h}$ of cisplatin nephrotoxicity, while HDAC1, HDAC2, HDAC3, HDAC6, Sirt3, and Sirt4 were induced at $72 \mathrm{~h}^{9}$. However, it remains unclear which $\mathrm{HDAC}(\mathrm{s})$ mediate cisplatin nephrotoxicity. Provided the role of HDAC in regulating acetylation, several ATG proteins may be subjected to the regulation by acetylation/deacetylation ${ }^{46,47}$. To understand how HDACis activate autophagy in proximal tubular cells, we examined the effects of TSA on AMPK and mTOR, two well-recognized autophagy regulator. We found that TSA could enhance AMPK activation, while blocking mTOR activation, in cisplatin nephrotoxicity (Fig. 10). In view of the opposite functions of AMPK and mTOR in autophagy regulation, it is suggested that HDACis may activate autophagy during cisplatin nephrotoxicity at least partially through AMPK and mTOR. 


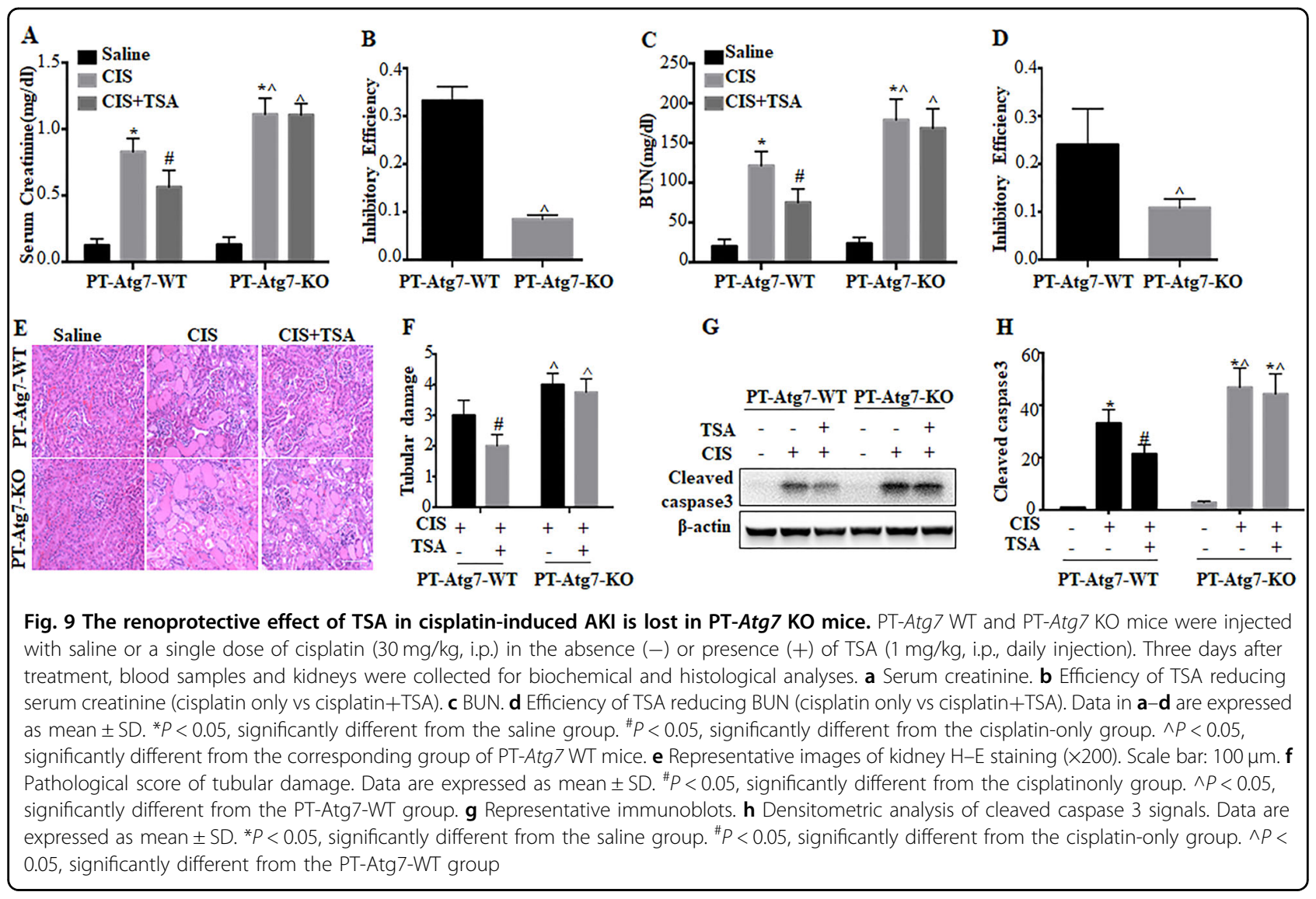

\section{Materials and methods}

\section{Reagents and antibodies}

TSA was purchased from Enzo Life Sciences. Carbobenzoxy-Asp-Glu-Val-Asp-7-amino-4-trifluoromethyl coumarin (DEVD-AFC) and 7-amino-4-trifluoromethyl coumarin (AFC) were from Enzyme Systems Products (Livermore, CA). Unless indicated, all other reagents including cisplatin and chloroquine were purchased from Sigma (St. Louis, MO). The following primary antibodies were used: anti-LC3B from Novus Biologicals (Littleton, $\mathrm{CO})$; anti-ATG7, anti- $\beta$-actin and anti-cyclophilin B from Abcam; and anti-cleaved caspase3, anti-AMPK, antiphospho-AMPK (Thr172), anti-P70S6K, anti-phosphoP70S6K (T389) from Cell Signaling Technology (Danvers, MA). All secondary antibodies for immunoblot analysis were from Thermo Scientific (Rockford, IL).

\section{Animals}

C57BL/6 mice were purchased from Jackson Laboratory (Bar Harbor, ME). Using Cre-loxP technology, a conditional KO mouse model with Atg7 specifically deleted from renal proximal tubules (PT-Atg7 $\mathrm{KO}$ ) was recently generated and characterized by our laboratory ${ }^{34,48}$. The autophagy reporter mice expressing RFP-GFP-LC3 transgene under the control of a CAG promoter (CAG-
RFP-GFP-LC3 mice) were originally provided by Dr. Joseph A. Hill at University of Texas Southwestern Medical Center (Dallas, TX) ${ }^{36}$. All animals were maintained in a pathogen-free facility at Charlie Norwood VA Medical Center under 12/12-h light/dark pattern with free access to water and food. All animal experiments were carried out according to a protocol approved by the Institutional Animal Care and Usage Committee in Charlie Norwood VA Medical Center.

\section{Mouse model of cisplatin-induced AKI and TSA treatment}

Male mice aged 8-12 weeks were used in this study. For cisplatin injury, mice were intraperitoneally (i.p.) injected with a single dose of cisplatin at $30 \mathrm{mg} / \mathrm{kg}$, while control animals were injected with a comparable volume of saline $^{49}$. To test the effect of chloroquine, $60 \mathrm{mg} / \mathrm{kg}$ chloroquine was injected (i.p.) $1 \mathrm{~h}$ prior to cisplatin administration and then daily after cisplatin treatment. To test the effect of TSA, $1 \mathrm{mg} / \mathrm{kg}$ TSA was injected (i.p.) 2 days prior to and daily after cisplatin administration.

Analysis of autophagy dynamics by a tandem mRFP-GFPLC3 reporter in transfected cells and in transgenic mice

The dynamic process of autophagy (autophagosome formation and maturation to autolysosomes) was 

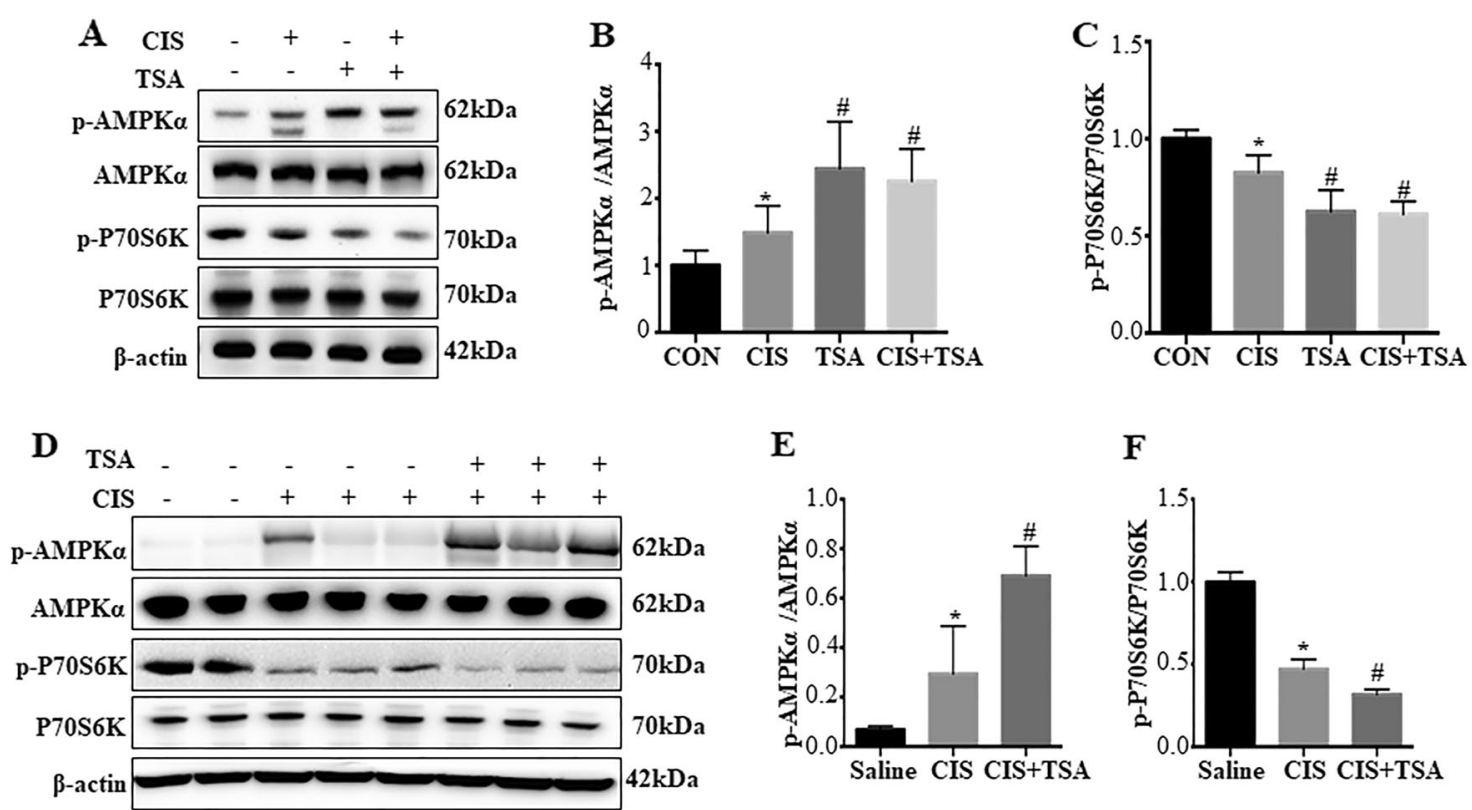

$\mathbf{E}$

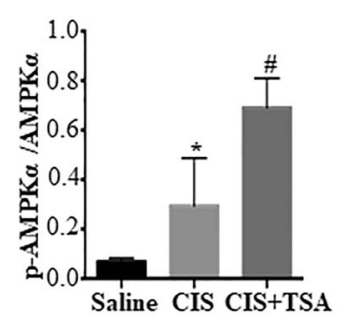

$\mathbf{F}$

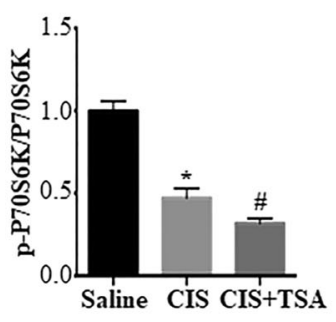

Fig. 10 TSA activates AMPK and inactivates mTOR during cisplatin treatment of RPTC cells and C57BI/6 mice. a-c RPTC cells were untreated or treated with cisplatin $(20 \mu \mathrm{M})$ only, TSA $(0.1 \mu \mathrm{M})$ only, or cisplatin+TSA for $20 \mathrm{~h}$. After treatment, cells were collected for immunoblot analysis of $\mathrm{p}$ AMPK, AMPK, p-P70S6K, and P70S6K. $\beta$-Actin was used as a loading control. a Representative immunoblots. $\mathbf{b}$, c Densitometric analysis of $p$-AMPK and $\mathrm{p}$-P70S6K signals after normalization with AMPK and P70S6K, respectively. Data are expressed as mean \pm SD. ${ }^{*} P<0.05$, significantly different from the control group. ${ }^{\#} P<0.05$, significantly different from the cisplatin-only group. $\mathbf{d}-\mathbf{f} C 57 \mathrm{BL} / 6$ mice were injected with saline or a single dose of cisplatin (30 mg/kg, i.p.) in the absence (-) or presence (+) of TSA (1 mg/kg, i.p., daily injection). Kidneys were collected 3 days after treatment for immunoblot analysis of p-AMPK, AMPK, p-P70S6K, and P70S6K. $\beta$-Actin was used as a loading control. d Representative immunoblots. e, $\mathbf{f}$ Densitometric analysis of $\mathrm{p}$-AMPK and p-P70S6K signals after normalization with total AMPK and P70S6K, respectively. Data are expressed as mean \pm SD. ${ }^{*} P<0.05$, significantly different from the saline group. ${ }^{\#} P<0.05$, significantly different from the cisplatin-only group

analyzed in cultured proximal tubular cells expressing mRFP-GFP-LC3 or in CAG-RFP-GFP-LC3 mice as described in our recent work $^{35}$. The rationale of this method is that acid-sensitive GFP is quenched in the low $\mathrm{pH}$ lysosomal environment, whereas acid-insensitive RFP is more stable and maintained. Thus co-localization of RFP fluorescence with GFP in a particle indicates an autophagosome, and a RFP-only signal is considered an autolysosome $\mathrm{e}^{36,50}$. For in vitro experiments, cultured proximal tubular cells were transiently transfected with mRFP-GFP-LC3 (ptfLC3, Addgene plasmid 21074). After treatment, the cells were fixed with $4 \%$ paraformaldehyde for fluorescence microscopy (Zeiss 780 upright confocal microscope). For quantitative analysis, approximately 100 transfected cells from 10 to 20 random fields $(\times 630)$ were analyzed in each condition. The numbers of GFP-LC3 puncta per cell and RFP-LC3 puncta per cell were counted separately using ImageJ. The number of autophagosomes was indicated by GFP dots and the number of autolysosomes was obtained by subtracting GFP dots from RFP dots. The number of autolysosomes was further divided by the total number of RFP dots to indicate the autophagic flux rate. For in vivo experiments, after treatment CAG-RFP-GFP-LC3 mice were perfused fixed with $4 \%$ paraformaldehyde. Kidneys were further fixed overnight with the same fixative, balanced with 30\% sucrose, and embedded in Optimal Cutting Temperature compound for cryo-section and confocal microscopy. For each section, 8-10 fields ( $\times 630)$ were selected randomly and quantitative analysis was performed by the method described above in cultured cells.

\section{Renal function}

Renal function was determined by BUN and serum creatinine measurements using commercial kits from Stanbio Laboratory (Boerne, TX). In brief, blood samples were collected for coagulation and centrifugation at room temperature to collect serum. For BUN, the reaction was conducted at $100{ }^{\circ} \mathrm{C}$ for $12 \mathrm{~min}$ and the absorbance at $520 \mathrm{~nm}$ was recorded by the end of reaction. For serum creatinine, samples were added to a pre-warmed $\left(37^{\circ} \mathrm{C}\right)$ reaction mixture and the absorbance at $510 \mathrm{~nm}$ was monitored kinetically at 20 and $80 \mathrm{~s}$ of reaction. BUN and creatinine levels $(\mathrm{mg} / \mathrm{dl})$ were then calculated based on standard curves.

\section{Histological examination}

Kidney tissues were harvested, fixed with $4 \%$ paraformaldehyde, embedded in paraffin, and sectioned at 4 
$\mu \mathrm{m} . \mathrm{H}-\mathrm{E}$ staining was performed using standard procedures and renal tubules with the following histopathological changes were considered injured: loss of brush border, tubular dilation and disruption, cast formation, and cell lysis. Tissue damage was examined in a blind manner and scored by the percentage of damaged tubules: 0 , no damage; $1,<25 \% ; 2,25-50 \%$; 3, 50-75\%; 4, >75\%.

\section{Immunohistochemical staining of LC3B}

Kidneys were fixed in $4 \%$ paraformaldehyde overnight and processed for routine embedding in paraffin. After rehydration, antigen retrieval was performed by incubation with $1 \mathrm{mM}$ EDTA ( $\mathrm{pH} 8.0$ ) at $95-100^{\circ} \mathrm{C}$ for $1 \mathrm{~h}$. After subsequently incubated with $0.03 \% \mathrm{H}_{2} \mathrm{O}_{2}$ to block endogenous peroxidase activity and with a buffer containing $2 \%$ bovine serum albumin, $0.2 \%$ milk, $2 \%$ normal donkey serum, and $0.8 \%$ Triton X-100 to reduce non-specific binding, the slides were exposed to anti-LC3B antibodies at $4{ }^{\circ} \mathrm{C}$ overnight. Negative controls were done by replacing the primary antibody with antibody diluent. After incubation with avidin-biotin blocking reagent (Vector Laboratories, SP-2001), the slides were exposed to 1:500 biotinylated donkey anti-rabbit secondary antibody (Millipore, AP) for $1 \mathrm{~h}$ at room temperature. Following signal amplification with Tyramide Signal Amplification Biotin System (Perkin Elmer, NEL700A001KT), the sections were incubated with a VECTASTAIN ${ }^{\circledR}$ ABC kit (Vector Laboratories, PK-6100) and signals were developed with a DAB kit (Vector Laboratories). For quantification of LC3B staining, $10-20$ fields $(\times 400)$ were randomly selected from each slide and the number of LC3B puncta per proximal tubule was evaluated using ImageJ.

\section{Cell lines}

Immortalized RPTC line was originally obtained from Dr. Ulrich Hopfer (Case Western Reserve University, Cleveland, $\mathrm{OH}$ ) and maintained in Dulbecco's Modified Eagle's Medium/F-12 medium supplemented with $10 \%$ fetal bovine serum and growth factors ${ }^{51}$. Stable floxed control (Atg7 WT) and Atg7 KO MPTC lines (MPTC) were generated in our recent study ${ }^{35}$. Briefly, primary kidney proximal tubular cells were isolated from 5 -weekold Atg7-loxP mice. The cells were cultured for immortalization by transfecting with SV40 larger T-antigen. The immortalized proximal tubular cells were then infected with retroviruses expressing Cre-PURO-IRES-GFP (pMSCV-Cre-PIG, Addgene plasmid 50395) or control viruses to establish stable $\operatorname{Atg} 7 \mathrm{WT}$ and $\operatorname{Atg} 7 \mathrm{KO}$ cell lines.

\section{Cisplatin and TSA treatment of RPTCs}

Cells were plated in 35-mm dishes to reach 90\% confluence by next day. To induce apoptosis, cells were incubated with $20 \mu \mathrm{M}$ cisplatin for $20 \mathrm{~h}$. TSA was given either alone at different concentrations $(0.05,0.1,0.2 \mu \mathrm{M})$ or together with cisplatin at a concentration of $0.1 \mu \mathrm{M}$. In some experiments, cells were also treated with cisplatin and TSA in the presence of $20 \mu \mathrm{M}$ chloroquine. After treatment, cells were monitored morphologically or harvested for biochemical analyses. For cell lysis, both floating and adherent cells were collected.

\section{Examination of apoptosis}

Apoptosis was examined by morphology and caspase activity by standard methods. Morphologically, cells were stained with $10 \mu \mathrm{g} / \mathrm{ml}$ Hoechst 33342 to examine cellular and nuclear morphology by phase contrast and fluorescence microscopy, respectively. For each condition, four fields with $\sim 200$ cells per field were randomly selected and cells with typically apoptotic characterizations were counted to estimate apoptosis percentage. To measure caspase activity, cells were extracted with $1 \%$ Triton X100 and $20 \mu \mathrm{g}$ protein were added to enzymatic reactions containing $50 \mu \mathrm{M}$ DEVD-AFC, a fluorogenic peptide substrate of caspases. After $1 \mathrm{~h}$ incubation at $37^{\circ} \mathrm{C}$, fluorescence was measured at excitation $360 \mathrm{~nm} / \mathrm{emission}$ $530 \mathrm{~nm}$. Based on a standard curve constructed with free $\mathrm{AFC}$, the fluorescence reading was converted into the nanomolar amount of liberated AFC per mg protein to indicate caspase activity.

\section{Immunoblot analysis}

Whole cell or tissue lysates from renal cortex and out medulla were extracted in $2 \%$ sodium dodecyl sulfate (SDS) buffer (62.5 mM Tris-HCl, pH 6.8, 2\% SDS, 10\% glycerol) containing protease inhibitor cocktail (SigmaAldrich) and Benzonase nuclease (EMD Millipore). Protein concentration was estimated with the BCA Protein Assay Kit (Thermo Scientific). Equal amounts of protein were loaded in each lane and separated by SDSpolyacrylamide gel electrophoresis. After transfer to polyvinylidene difluoride membranes, blots were blocked in $5 \%$ milk, probed with primary and secondary antibodies, and visualized with an enhanced chemiluminescence kit. Either $\beta$-actin or cyclophilin $B$ was used to monitor protein loading and transferring.

\section{Statistics}

Qualitative data including immunoblots and cell images are representatives of at least three experiments. Quantitative data were expressed as means \pm SD. Statistical analysis was conducted using the GraphPad Prism software. Statistical differences in multiple groups were determined by multiple comparisons with analysis of variance followed by Tukey's post-tests. Statistical differences between two groups were determined by two-tailed unpaired or paired Student's $t$-test. $P<0.05$ was considered significantly different. 


\section{Acknowledgements}

The authors were partly supported by the grants from National Natural Science Foundation of China (81720108008, 81430017), China Scholarship Council, the National Institutes of Health of USA, and Department of Veterans Administration of USA.

\section{Author details}

'Department of Nephrology, The Second Xiangya Hospital, Central South University, Changsha, Hunan 410011, China. ${ }^{2}$ Department of Cellular Biology \& Anatomy, Medical College of Georgia at Augusta University and Charlie Norwood VA Medical Center, Augusta, GA 30912, USA. ${ }^{3}$ Department of Pharmacology \& Toxicology, Medical College of Georgia at Augusta University and Charlie Norwood VA Medical Center, Augusta, GA 30912, USA.

${ }^{4}$ Department of Pathology and Laboratory Medicine, Indiana University School of Medicine, Indianapolis, IN, USA

\section{Conflict of interest}

The authors declare that they have no conflict of interest.

\section{Publisher's note}

Springer Nature remains neutral with regard to jurisdictional claims in published maps and institutional affiliations.

Supplementary Information accompanies this paper at https://doi.org/ 10.1038/s41419-018-0374-7.

Received: 25 October 2017 Revised: 23 January 2018 Accepted: 5 February 2018

Published online: 23 February 2018

\section{References}

1. Seto, E. \& Yoshida, M. Erasers of histone acetylation: the histone deacetylase enzymes. Cold Spring Harb. Perspect. Biol. 6, a018713 (2014).

2. Falkenberg, K. J. \& Johnstone, R. W. Histone deacetylases and their inhibitors in cancer, neurological diseases and immune disorders. Nat. Rev. Drug Discov. 13, 673-691 (2014)

3. Li, Y. \& Seto, E. HDACs and HDAC inhibitors in cancer development and therapy. Cold Spring Harb. Perspect. Med. 6, pii: a026831 (2016).

4. Pang, M. et al. Inhibition of histone deacetylase activity attenuates renal fibroblast activation and interstitial fibrosis in obstructive nephropathy. Am. J. Physiol. Ren. Physiol. 297, F996-F1005 (2009).

5. Liu, N. et al. Blocking the class I histone deacetylase ameliorates renal fibrosis and inhibits renal fibroblast activation via modulating TGF-beta and EGFR signaling. PLOS ONE 8, e54001 (2013).

6. Levine, M. H. et al. Class-specific histone/protein deacetylase inhibition protects against renal ischemia reperfusion injury and fibrosis formation. Am. J. Transplant. 15, 965-973 (2015).

7. Arany, I., Herbert, J., Herbert, Z. \& Safirstein, R. L. Restoration of CREB function ameliorates cisplatin cytotoxicity in renal tubular cells. Am. J. Physiol. Ren. Physiol. 294, F577-F581 (2008).

8. Dong, G., Luo, J., Kumar, V. \& Dong, Z. Inhibitors of histone deacetylases suppress cisplatin-induced p53 activation and apoptosis in renal tubular cells. Am. J. Physiol. Ren. Physiol. 298, F293-F300 (2010).

9. Ranganathan, P. et al. Histone deacetylase-mediated silencing of AMWAP expression contributes to cisplatin nephrotoxicity. Kidney Int. 89, 317-326 (2016).

10. Cao, S., Zhong, B., Yue, H., Zeng, H. \& Zeng, J. Development and testing of a sustainable environmental restoration policy on eradicating the poverty trap in China's Changting County. Proc. Natl. Acad. Sci. USA 106, 10712-10716 (2009).

11. Fan, L. X., Li, X., Magenheimer, B., Calvet, J. P. \& Li, X. Inhibition of histone deacetylases targets the transcription regulator $1 \mathrm{~d} 2$ to attenuate cystic epithelial cell proliferation. Kidney Int. 81, 76-85 (2012).

12. Gilbert, R. E. et al. Histone deacetylase inhibition attenuates diabetesassociated kidney growth: potential role for epigenetic modification of the epidermal growth factor receptor. Kidney Int. 79, 1312-1321 (2011).
13. Wang, X. et al. Histone deacetylase 4 selectively contributes to podocyte injury in diabetic nephropathy. Kidney Int. 86, 712-725 (2014).

14. Zhong, Y. et al. Renoprotective effect of combined inhibition of angiotensinconverting enzyme and histone deacetylase. J. Am. Soc. Nephrol. 24, 801-811 (2013).

15. Brilli, L. L., Swanhart, L. M., de Caestecker, M. P. \& Hukriede, N. A. HDAC inhibitors in kidney development and disease. Pediatr. Nephrol. 28, 1909-1921 (2013).

16. Liu, N. \& Zhuang, S. Treatment of chronic kidney diseases with histone deacetylase inhibitors. Front. Physiol. 6, 121 (2015).

17. Dong, G. et al. Induction of apoptosis in renal tubular cells by histone deacetylase inhibitors, a family of anticancer agents. J. Pharmacol. Exp. Ther. 325, 978-984 (2008)

18. dos Santos, N. A., Carvalho Rodrigues, M. A., Martins, N. M. \& dos Santos, A. C. Cisplatin-induced nephrotoxicity and targets of nephroprotection: an update. Arch. Toxicol. 86, 1233-1250 (2012).

19. Miller, R. P., Tadagavadi, R. K., Ramesh, G. \& Reeves, W. B. Mechanisms of cisplatin nephrotoxicity. Toxins (Basel) 2, 2490-2518 (2010).

20. Pabla, N. \& Dong, Z. Cisplatin nephrotoxicity: mechanisms and renoprotective strategies. Kidney Int. 73, 994-1007 (2008).

21. Yan, M., Tang, C., Ma, Z., Huang, S. \& Dong, Z. DNA damage response in nephrotoxic and ischemic kidney injury. Toxicol. Appl. Pharmacol. 313, 104-108 (2016).

22. Zhu, S., Pabla, N., Tang, C., He, L. \& Dong, Z. DNA damage response in cisplatininduced nephrotoxicity. Arch. Toxicol. 89, 2197-2205 (2015).

23. Linkermann, A. et al. Regulated cell death in AKI. J. Am. Soc. Nephrol. 25 2689-2701 (2014).

24. Ohsumi, Y. Historical landmarks of autophagy research. Cell Res. 24, 9-23 (2014).

25. Yang, Z. \& Klionsky, D. J. Eaten alive: a history of macroautophagy. Nat. Cell Biol. 12, 814-822 (2010).

26. Choi, A. M., Ryter, S. W. \& Levine, B. Autophagy in human health and disease. N. Engl. J. Med. 368, 651-662 (2013).

27. Huber, T. B. et al. Emerging role of autophagy in kidney function, diseases and aging. Autophagy 8, 1009-1031 (2012).

28. Livingston, M. J. \& Dong, Z. Autophagy in acute kidney injury. Semin. Nephrol. 34, 17-26 (2014).

29. Kaushal, G. P. \& Shah, S. V. Autophagy in acute kidney injury. Kidney Int. 89 779-791 (2016).

30. Havasi, A. \& Dong, Z. Autophagy and tubular cell death in the kidney. Semin. Nephrol. 36, 174-188 (2016).

31. Tang, C., et al. PINK1-PRKN/PARK2 pathway of mitophagy is activated to protect against Renal ischemia-reperfusion injury. Autophagy 1-45 (2017).

32. Klionsky, D. J. et al. Guidelines for the use and interpretation of assays for monitoring autophagy (3rd edition). Autophagy 12, 1-222 (2016).

33. Periyasamy-Thandavan, $\mathrm{S}$. et al. Autophagy is cytoprotective during cisplatin injury of renal proximal tubular cells. Kidney Int. 74, 631-640 (2008).

34. Jiang, M. et al. Autophagy in proximal tubules protects against acute kidney injury. Kidney Int. 82, 1271-1283 (2012).

35. Livingston, M. J. et al. Persistent activation of autophagy in kidney tubular cells promotes renal interstitial fibrosis during unilateral ureteral obstruction. Autophagy 12, 976-998 (2016).

36. Li, L., Wang, Z. V., Hill, J. A. \& Lin, F. New autophagy reporter mice reveal dynamics of proximal tubular autophagy. J. Am. Soc. Nephrol. 25, 305-315 (2014).

37. Wong, P. M., Puente, C., Ganley, I. G. \& Jiang, X. The ULK1 complex: sensing nutrient signals for autophagy activation. Autophagy 9, 124-137 (2013).

38. Cheng, H., Fan, X., Lawson, W. E., Paueksakon, P. \& Harris, R. C. Telomerase deficiency delays renal recovery in mice after ischemia-reperfusion injury by impairing autophagy. Kidney Int. 88, 85-94 (2015).

39. Patschan, D., Schwarze, K, Henze, E., Patschan, S. \& Muller, G. A. Endothelial autophagy and endothelial-to-mesenchymal transition (EndoMT) in eEPC treatment of ischemic AKI. J. Nephrol. 29, 637-644 (2016).

40. Khan, S., Kumar, S. \& Jena, G. Valproic acid reduces insulin-resistance, fat deposition and FOXO1-mediated gluconeogenesis in type-2 diabetic rat. Biochimie 125, 42-52 (2016).

41. Xie, M. et al. Histone deacetylase inhibition blunts ischemia/reperfusion injury by inducing cardiomyocyte autophagy. Circulation 129, 1139-1151 (2014).

42. Shao, A. et al. Enhancement of autophagy by histone deacetylase inhibitor trichostatin $\mathrm{A}$ ameliorates neuronal apoptosis after subarachnoid hemorrhage in rats. Mol. Neurobiol. 53, 18-27 (2016). 
43. Kim, M. S. et al. Inhibition of histone deacetylase increases cytotoxicity to anticancer drugs targeting DNA. Cancer Res. 63, 7291-7300 (2003)

44. Fahmi, T. et al. Mechanism of graphene-induced cytotoxicity: role of endonucleases. J. Appl. Toxicol. 37, 1325-1332 (2017).

45. Jang, D. S. et al. Novel cytoprotective inhibitors for apoptotic endonuclease G. DNA Cell. Biol. 34, 92-100 (2015).

46. Lapierre, L. R., Kumsta, C., Sandri, M., Ballabio, A. \& Hansen, M. Transcriptional and epigenetic regulation of autophagy in aging. Autophagy 11,867-880 (2015).

47. Xie, Y. et al. Posttranslational modification of autophagy-related proteins in macroautophagy. Autophagy 11, 28-45 (2015)
48. Yi, M. et al. Autophagy is activated to protect against podocyte injury in adriamycin-induced nephropathy. Am. J. Physiol. Ren. Physiol. 313, F74-F84 (2017).

49. Zhang, D. et al. Tubular p53 regulates multiple genes to mediate AKI. J. Am Soc. Nephrol. 25, 2278-2289 (2014).

50. Kimura, S., Noda, T. \& Yoshimori, T. Dissection of the autophagosome maturation process by a novel reporter protein, tandem fluorescent-tagged LC3. Autophagy 3, 452-460 (2007).

51. Woost, P. G. et al. Immortalization and characterization of proximal tubule cells derived from kidneys of spontaneously hypertensive and normotensive rats. Kidney Int. 50, 125-134 (1996). 